\title{
The SW-MSA Calculation of Self-Diffusion Coefficients in Liquid Lithium and Rubidium
} Acta Physica Polonica A 129, 310 (2016), ERRATUM

\author{
N.E. Dubinin \\ Ural Federal University, 19 Mira Str., 620002 Ekaterinburg, Russia \\ and \\ Institute of Metallurgy of the Ural Division of the Russian Academy of Sciences, \\ 101 Amundsen Str., 620016 Ekaterinburg, Russia
}

\begin{abstract}
Successfully used recently for liquid $\mathrm{Na}$ and $\mathrm{K}$ the approach combined the linear trajectory approximation with the square-well model in the semi-analytical representation of the mean spherical approximation to calculate the self-diffusion coefficients is applied here to liquid $\mathrm{Li}$ and $\mathrm{Rb}$. As well as earlier for $\mathrm{Na}$ and $\mathrm{K}$, the results obtained are found to be in reasonable agreement with the available experimental data and confirm previous conclusion that the square-well model within the mean spherical approximation is quite useful for description of diffusion properties in liquid alkali metals.
\end{abstract}

original DOI: 10.12693/APhysPolA.129.884

actual DOI: 10.12693/APhysPolA.135.553

PACS/topics: 61.20.Gy, 61.20.Ne, 61.25.Mv, 66.10.C-

This article was originally published on March 2016 with some mistakes:

1. In the right-hand side of Eq. (5), the mean atomic density, $\rho$, was omitted. In corrected form this equation must be written as follows:

$$
\xi_{2}^{\mathrm{SW}-\mathrm{MSA}-\mathrm{SA}}=-\rho \frac{(\beta \pi M)^{1 / 2}}{12 \pi^{2}} \int_{0}^{\infty}\left(S_{\mathrm{SW}-\mathrm{MSA}-\mathrm{SA}}(q)-1\right) \varphi_{\mathrm{SW}}(q) q^{3} \mathrm{~d} q .
$$

2. The correct caption of Table II is "Self-diffusion coefficient, $D \times 10^{9}\left[\mathrm{~m}^{2} / \mathrm{s}\right]$ " instead of "Self-diffusion coefficient, $D \times 10^{-9}\left[\mathrm{~m}^{2} / \mathrm{s}\right] "$

3. The sentence "The semi-analytical representation of the mean spherical approximation is postulated as follows: (...)" placed before Eq. (10) has been changed to: "The semi-analytical representation of the mean spherical approximation for the SW fluid is postulated as follows [4]: (...)" for more accuracy and clarity.

Author apologizes for these errors.

corresponding author; e-mail: ned67@mail.ru 